\title{
COMPARATIVE FEASIBILITY OF DOBUTAMINE STRESS ECHOCARDIOGRAPHY PERFORMED WITH AND WITHOUT INTRAVENOUS CONTRAST IN PATIENTS WITH CLASS III OBESITY
}

\author{
Carl Carlino ${ }^{1}$, Saravanan Kuppuswamy ${ }^{1}$, Lerin McCray $^{1}$, Kul Aggarwal ${ }^{1}$, and Martin \\ Alpert $^{2}$ \\ ${ }^{1}$ University of Missouri School of Medicine \\ ${ }^{2}$ Affiliation not available
}

July 2, 2021

\begin{abstract}
Background: Concern exists regarding adequacy of visualization of stress echocardiograms performed without intravenous contrast in persons with Class III obesity (body mass index [?] $40 \mathrm{~kg} / \mathrm{m} 2$ ). Methods: Dobutamine stress echocardiography (DSE) was performed on 128 candidates for bariatric surgery with class III obesity without chest pain or pre-existent coronary artery disease (CAD). DSE without intravenous contrast was initially performed on 62 patients with class III obesity, then was subsequently was performed with intravenous contrast on 66 patients with class III obesity. Left ventricular (LV) regional wall motion was assessed at baseline and peak stress using the 16-segment model. Results: In the intravenous contrast group 1046 of $1056 \mathrm{LV}$ segments studied (99.1\%) were well-visualized and interpretable at baseline and 1044 of 1056 LV segments studied (98.9\%) were well-visualized and interpretable at peak stress. In the non-contrast group 905 of 992 segments studied (91.2\%) were well-visualized and interpretable at baseline and 886 of 992 segments studied (89.3\%) were well-visualized and interpretable at peak stress. A significantly greater number of LV segments were well-visualized and interpretable in the intravenous contrast group than in the group compared to the non-contrast group, at baseline and at peak stress ( $<<0.00001$ for both). DSE was positive for ischemia in 1 patient. All patients underwent bariatric surgery without cardiovascular complications. Six months after surgery, all patients were alive; none developed cardiovascular events. Conclusion: The use of intravenous contrast during DSE significantly improves visualization and interpretability of LV segments in patients with class III obesity.
\end{abstract}

\section{INTRODUCTION}

Obesity, particularly central obesity is commonly associated with a variety of traditional and non-traditional cardiovascular (CV) risk factors and is a key component of the metabolic syndrome which itself is a potent risk factor for cardiovascular disease (CVD). ${ }^{1-3}$ Accordingly, obesity is considered to be a risk factor for CVD. ${ }^{1-3}$ Moreover, the $27^{\text {th }}$ Bethesda Conference classified obesity as an independent risk factor for CVD. ${ }^{2}$ For these reasons testing for CVD, particularly coronary artery disease (CAD) is performed frequently in both symptomatic and asymptomatic patients with obesity, often in preparation for elective non-cardiac surgery. ${ }^{4}$ In patients with mildly-to-moderate obesity, standard methods of stress testing can be performed with an acceptable sensitivity, specificity, and predictive accuracy. ${ }^{5-9}$ In patients with extreme (Class III) obesity there are limitations associated with standard stress testing modalities. ${ }^{5-9}$ Poor functional capacity may limit the effectiveness of stress tests that employ treadmill exercise. ${ }^{3,5,7-9}$ Excessive attenuation and weight restrictions may interfere with the ability to obtain accurate pharmacologic myocardial perfusion scans. ${ }^{7,9}$ Weight and size limitations and cost considerations cause stress cardiac magnetic resonance imaging 
to be used infrequently in patients with class III obesity. ${ }^{7}$ Dobutamine stress echocardiography (DSE) is the most commonly-used stress testing modality in such patients. ${ }^{5,7,9,10}$ However, concern exists about the ability to adequately image left ventricular (LV) segments without the use of intravenous contrast. The purpose of this prospective non-randomized study was to compare feasibility of DSE with and without intravenous contrast in patients with class III obesity without chest pain or pre-existent CAD who were candidates for and eventually underwent bariatric surgery

Alpert

\section{SUBJECTS AND METHODS}

\section{Subjects}

Consecutive adult patients with class III obesity who were candidates for bariatric surgery were considered for entry into the study. Class III obesity was defined as body mass index [?] $40 \mathrm{~kg} / \mathrm{m}^{2}{ }^{8}$ Patients $<18$ years of age, those with a history of chest pain within 6 months of evaluation, and patients with an established history of CVD (other than systemic hypertension) including CAD were excluded from the study. Patients with current hyperthyroidism or hypothyroidism, severe bradycardia $(<40$ beats $/ \mathrm{min})$, and those with a resting heart rate $>110$ beats/min, including those with supraventricular tachyarrhythmias and ventricular tachycardia and patients with a blood pressure [?] 180/110 $\mathrm{mmHg}$ on multiple encounters were excluded from the study.

\section{Methods}

All patients were evaluated by a cardiologist. Each patient received a complete medical history and physical examination. A 12-lead resting electrocardiogram, chest x-ray, comprehensive metabolic panel, fasting lipid panel, urinalysis, complete blood count, and a serum thyroid stimulating hormone were performed on or obtained from all patients. Electrolyte abnormalities, if present, were corrected prior to electrocardiography and stress testing.

At the time of this study, DSE was the preferred stress testing modality by our bariatric surgeons. DSE was performed in accordance with American Society of Echocardiography recommendations. ${ }^{9,10}$ Following acquisition of baseline images, dobutamine was administered by IV infusion beginning with a dose of 5 $\mathrm{mcg} / \mathrm{kg} / \mathrm{min}$. The dose was increased at 3 -minute

Alpert

intervals in a graded fashion from 5 to 10 to 20 to $40 \mathrm{mcg} / \mathrm{kg} / \mathrm{min}$. Blood pressure, heart rate, and 12 lead electrocardiograms were recorded and echocardiographic images were obtained at baseline and during the third minute of dobutamine infusion for each dose. There was continuous electrocardiographic rhythm monitoring. The test was terminated after data acquisition at the dose of dobutamine that produced a heart rate [?] $85 \%$ of the predicted (target) heart rate or following infusion of the $40 \mathrm{mcg} / \mathrm{kg} / \mathrm{min}$ dose. If [?] $85 \%$ of target heart rate was not achieved with infusion of the peak dose of dobutamine, atropine sulfate was administered by bolus IV injection during the final stage starting with a dose of $0.5 \mathrm{mg}$ followed by subsequent doses of $0.5 \mathrm{mg}$ every 5 minutes until [?] $85 \%$ of target heart rate was achieved or until the patient received a total dose of $2.0 \mathrm{mg}$.

Due to an extended pause in the commercial availability of the IV contrast-enhancing agent Optison, our medical system relied solely on non-contrast images with DSE during the period of unavailability of Optison. During this period 62 consecutive eligible patients with class III obesity were entered into the study and underwent DSE without intravenous contrast injection. Subsequently, when the use of intravenous contrast agents was deemed safe for use during DSE, 66 consecutive eligible patients underwent DSE with intravenous contrast injection. With the subsequent availability of Definity (Perfluten Lipid Micrspheres, Lantheus Medical Imaging, North Billerca, MA), intravenous contrast injection resumed. Intravenous contrast injection consisted of bolus IV infusion of Definity. The dose was $10 \mathrm{microL} / \mathrm{kg}$, agitated and injected over 30-60 seconds and followed by a $10 \mathrm{~mL}$ normal saline flush. In patients who received Definity, bolus infusion occurred 
just prior to acquisition of baseline echocardiographic images. As previously noted, patients enrolled in this prospective study were not randomized. No

Alpert

attempt was made to match patients who received IV contrast with those who did not receive IV contrast.

Transthoracic 2-dimensional echocardiographic images were obtained using a General Electric Vivid E9 echocardiograph with a GE M5S-DM 1.5-4.5 MHz transducer probe with second harmonic capability. Images were arranged in a quad screen format. LV regional wall motion was assessed in accordance with American Society of Echocardiography guidelines. ${ }^{9,10}$ Sixteen LV segments visualized in 4 planes were evaluated for evidence of myocardial ischemia as follows: Parasternal or apical long axis (mid-anteroseptal, basal anteroseptal, basal posterior, mid-inferolateral), parasternal short axis (mid-anterior, mid-anteroseptal, mid septal, mid- inferior, mid-inferolateral, mid-lateral), apical 4-chamber (apical septal, mid-septal, basal septal, basal lateral, mid-lateral, apical lateral), and-apical 2-chamber (apical anterior, mid-anterior, basal anterior, apical inferior, mid-inferior, basal inferior). ${ }^{9,11}$ Myocardial ischemia was diagnosed when normal LV regional wall motion segments at baseline transformed into dobutamine-induced hypokinesis, akinesis, or dyskinesis, or if there was dobutamine-induced global LV cavity dilatation and reduction of LV systolic function and failure of LV myocardial segment(s) to thicken during dobutamine stress. ${ }^{9-11}$ Individual regional LV segments were assessed at baseline and at peak stress. Individual LV segments were classified as "well-visualized and interpretable" or "poorly-visualized and not interpretable" ${ }^{9-11}$ Well-visualized LV segments were those in which the endocardial border was well-defined and interpretable for echocardiographic signs of myocardial ischemia. LV segments that were poorly-visualized included those in which the subendocardial border in part was poorly-defined or non-visualized

Alpert

and not interpretable for echocardiographic signs of myocardial ischemia. The numbers of LV segments that were well-visualized and interpretable for echocardiographic signs of myocardial ischemia in the group that received intravenous contrast and in the group that did not receive intravenous contrast were recorded and reported.

This study was not designed to assess long-term CV outcomes. Nevertheless, we cataloged CV outcomes during DSE (regional LV wall motion abnormalities, hypertension, hypotension, arrhythmias) and 6 months after bariatric surgery (all-cause and CV deaths, angina pectoris, myocardial infarction, stroke).

All procedures involving human participants in this study were in accordance with the ethical standards of the 1964 Helsinki declaration and its later amendments and comparable ethical standards. Informed written consent was obtained from all patients participating in this study. This study was approved by the University of Missouri-Columbia School of Medicine Institutional Review Board.

\section{Statistical Methods}

The chi square test was used to determine if significant differences existed in the proportion of categorical variables between groups receiving and not receiving intravenous contrast and between categorical variables within these groups. Student's "t" test for unpaired data was used to compare mean values for continuous variables between the group that received IV contrast and the group that did not receive intravenous contrast. LV regional wall motion was assessed by 2 investigators who were experienced echocardiographers (CC and MA).

Alpert

Differences were resolved by consensus. A p value $<0.05$ was required for statistical significance.

\section{RESULTS}

\section{Patient Characteristics}


A total of 132 patients were initially considered for entry into the study. Four patients were excluded because no LV endocardial borders could be visualized with or without use of intravenous contrast. Thus, the final study population consisted of 128 subjects including 62 patients who did not receive intravenous contrast and 66 patients who received intravenous contrast prior to baseline image acquisition. Table I shows patient characteristics for the study population as a whole and for the groups receiving and not receiving intravenous contrast.

\section{Feasibility of DSE with and without Intravenous Contrast Enhancement}

Table II shows the number of well-visualized and interpretable regional LV segments in the group of patients who received intravenous contrast and in the group of patients who did not receive intravenous contrast. Each LV plane is accompanied by the number of segments that were visualized well enough to assess for myocardial ischemia. The associated $\mathrm{p}$ value indicates whether the differences between the proportion of well-visualized and interpretable LV segments between the 2 groups was statistically significant, both at baseline and at peak stress. A total of $1056 \mathrm{LV}$ segments were evaluated in the group that received intravenous contrast. Of these, the number of segments that were well-visualized and interpretable was $1046(99.1 \%)$ at baseline and $1044(98.9 \%)$ at peak stress $(\mathrm{p}=0.975)$. A total of $992 \mathrm{LV}$ segments were evaluated in the group that did not receive intravenous contrast. Of these, the number of segments were well-

Alpert

visualized and interpretable were $905((91.6 \%)$ at baseline and $886(89.3 \%)$ at peak stress $(\mathrm{p}=0.745)$. The proportion of well-visualized and interpretable segments in the group that received intravenous contrast was significantly higher than that of the group that did not receive intravenous contrast, both at baseline $(\mathrm{p}<$ $0.00001)$ and during peak stress $(\mathrm{p}<0.00001)$. Poorly-visualized uninterpretable images in [?] 2 segments were present in 1 of the 66 patients who received intravenous contrast and in 8 of the 62 patients who did not receive intravenous contrast $(\mathrm{p}<0.02)$.

\section{Outcomes During DSE}

None of the 128 patients studied had regional wall motion abnormalities prior to dobutamine infusion. All patients achieved [?] $85 \%$ of target heart rate. One patient was found to have dobutamine-induced hypokinesis of mid-anterior and mid anteroseptal LV segments in the group that did not receive intravenous contrast. No dobutamine-induced regional wall motion abnormalities were noted in the group that received intravenous contrast enhancement. Coronary angiography was performed on the patient with a positive stress test and showed high-grade stenosis of the mid-portion of the left anterior descending coronary artery. Drugeluting stent placement was performed without incident. No patient in the group that received intravenous contrast had DSE findings suggestive of myocardial ischemia. No patient had dobutamine-induced global LV dilatation or systolic dysfunction or a hyper-dynamic response with LV outflow tract obstruction. No patient developed severe hypertension. Two patients developed mild transient hypotension that resolved spontaneously, 1 in each group $(\mathrm{p}=0.965)$. Isolated atrial and ventricular premature beats occurred in multiple patients in both the intravenous contrast and non-intravenous contrast groups. There were no episodes of ventricular tachycardia, atrial fibrillation, or other

Alpert

supraventricular tachyarrhythmias. Three patients required intravenous metoprolol due to persistent sinus tachycardia after completion of the DSE protocol.

\section{Outcomes Six Months After Bariatric Surgery}

All patients undergoing DSE eventually underwent bariatric surgery. Surgical procedures performed included laparoscopic adjustable gastric banding in $68 \%$, vertical sleeve gastrectomy in $11 \%$, vertical banded gastroplasty in $10 \%$, Roux-en-Y gastric bypass in $6 \%$, and biliopancreatic diversion with or without duodenal switch in 5\%. There were no CV complications during surgery or in the peri-operative period. All patients were received follow-up in-person 6 months after surgery. None were lost to follow-up. Based on an 
interim history, physical examination and a 12-lead electrocardiogram performed during the follow-up visit, no patients developed CV symptoms or complications.

\section{DISCUSSION}

This is the first study in patients with class III obesity to directly compare the feasibility and interpretability of DSE with and without the use of intravenous contrast enhancement, both cumulatively and on a segment-by-segment basis. ${ }^{13-19}$ The results indicate that in patients with class III obesity, DSE performed using intravenous contrast enhancement is superior to DSE performed without intravenous enhancement, particularly with regard to visualization of the lateral and anterior segments. Moreover, DSE without intravenous contrast was associated with a significantly higher frequency of poor- or non-visualization of [?] 2 segments (rendering them uninterpretable) than DSE performed with intravenous contrast. Our study consisted of patients with class III obesity without chest pain and without previously documented CAD. Most of our

Alpert

patients had at least 1 traditional CV risk factor and many had $>1$ risk factor. Of the 128 patients studied, 55 were diabetic. Thus, our study population simulated a group of patients with class III obesity who may be referred for stress testing prior to bariatric surgery to screen for myocardial ischemia in the absence of chest pain or documented pre-existent CAD. This comprises the majority of such referrals. Our results further suggest that myocardial ischemia is extremely uncommon in such patients.

Nine prior studies have evaluated patients with various degrees of obesity with transthoracic exercise stress echocardiography (ESE) or DSE to determine the feasibility of these studies in this population (4 studies), to assess prognosis based on the presence or absence of myocardial ischemia and other CV outcomes (8 studies). ${ }^{12-18}$ or both (3 studies). Lerakis et al studied 611 patients whose BMI was [?] $40 \mathrm{~kg} / \mathrm{m}^{2}$ to determine the feasibility of DSE prior to and the short- and long-term outcomes following bariatric surgery based on the results of the stress test. ${ }^{12}$ The study population consisted of $86.6 \%$ women and $13.4 \%$ men (mean age: $42 \pm 10$ years). The mean BMI was $48.0 \pm 6.1 \mathrm{~kg} / \mathrm{m}^{2}$ and mean body weight was $136 \pm 18 \mathrm{~kg}$. The end-points were all-cause mortality and multiple adverse cardiac events (cardiac death, acute coronary syndrome, urgent coronary revascularization) at 30 days and at 6 months following surgery. Adequate baseline echocardiographic images were achieved in 590 patients (96.6\%). Intravenous contrast was used in $72.2 \%$ of cases. Those without adequate baseline images were referred for other procedures to assess for myocardial ischemia. DSE was negative in 545 patients $(92.4 \%$ ), was inconclusive in $6.4 \%$, and was positive in 1 patient $(1.2 \%)$. Laparoscopic surgery was performed in $77 \%$ of cases. There were 3 early deaths (all from sepsis). There were no major cardiac events within 30 days of surgery. One patient developed acute coronary

Alpert

syndrome between 30 days and 6 months. Shah and colleagues reported the results of the multicenter Study of Ultrasound in Morbid Obesity (SUMO). ${ }^{13}$ Morbid obesity was defined as a BMI [?] $35 \mathrm{~kg} / \mathrm{m}^{2}$. The study population consisted of 209 subjects who were referred for evaluation of suspected CAD. The mean BMI was $39.3+-4.1 \mathrm{~kg} / \mathrm{m}^{2}$. ESE was performed in $60 \%$ and DSE in $40 \%$ of subjects. Intravenous contrast was used in $96 \%$ of patients. Mean follow-up was 13 months. Outcomes studied were all-cause mortality, myocardial infarction, and late coronary revascularization. Inducible ischemia was detected in 32 patients (15\%). Coronary angiography was performed in 25 patients. Twenty-two had obstructive coronary artery stenotic lesions in distributions that corresponded with the location of myocardial ischemia during ESE or DSE and $77 \%$ of whom received coronary revascularization. Predictors of the outcomes were an LV ejection fraction $<50 \%$ and inducible ischemia. Supariwala and colleagues retrospectively studied 652 consecutive obese patients (BMI [?] $30 \mathrm{~kg} / \mathrm{m}^{2}$ ) with multiple CV risk factors who underwent stress echocardiography. ${ }^{14}$ DSE was performed on 423 patients $(65 \%)$ and $229(35 \%)$ underwent ESE. The study population consisted of $84 \%$ women (mean age: $47+-10$ years). Mean BMI was $47+-9 \mathrm{~kg} / \mathrm{m}^{2}$ and mean body weight was 280 +- 69 pounds. The study population was $12 \%$ Caucasian, $56 \%$ Hispanic, and $30 \%$ African-American. Mean follow-up was $3.2+-2.7$ years. The studies were classified as technically difficult in 293 patients (45\%) and 
intravenous contrast was used in 229 subjects (35\%). Target heart rate was achieved in 553 patients (85\%). The test was considered suboptimal in 64 patients (10\%). During the follow-up period, there were 8 deaths. Deaths occurred in 1 patient with obesity (BMI: $30.0-39.9 \mathrm{~kg} / \mathrm{m}^{2}$ ), in 3 patients with extreme obesity (BMI: $40.0-49.9 \mathrm{~kg} / \mathrm{m}^{2}$ ), and in 4 patients with super-obesity (BMI [?] $50 \mathrm{~kg} / \mathrm{m}^{2}$ ). Hu and co-workers studied 62 patients who were overweight or obese (BMI 26-33

Alpert

$\left.\mathrm{kg} / \mathrm{m}^{2}\right) .{ }^{15}$ DSE was performed with and without intravenous contrast injection. Visualization of LV segments was scored as 0 (not-visualized), 1 (poorly-visualized), and 2 (well-visualized). A total of 992 segments were assessed. Each patient served as their own control with regard to acquisition of images with and without intravenous contrast enhancement (this process was not clearly-described). Compared to non-utilization of intravenous contrast, use of intravenous contrast was associated with higher sensitivity (82\% vs. $70 \%$ ), specificity ( $78 \%$ vs. $67 \%$ ), and accuracy ( $81 \%$ vs. $69 \%$ ). Visualization scores without contrast were 0 in 179 segments (18\%), 1 in 328 segments (38\%), and 2 in 433 segments (44\%). In the group that received intravenous contrast visualization scores were 0 were in 0 segments, 1 in 50 segments (5\%) and 2 in 942 segments (95\%). Silviera and colleagues performed physical stress echocardiography on 945 obese and 3105 non-obese patients. ${ }^{16}$ No significant differences were noted in the incidence of myocardial ischemia in obese patients $(19.0 \%)$ compared to non-obese patients (17.9\%). Logistic regression analysis identified age, female gender, diabetes mellitus, and hypertension, but not obesity as predictors of myocardial ischemia. Murphy et al performed a retrospective chart review on 704 patients to determine the prognostic value of a normal stress echocardiogram in overweight and obese subjects. ${ }^{17}$ The study population consisted of 366 obese patients (BMI [?] $30 \mathrm{~kg} / \mathrm{m}^{2}$ ), 196 overweight subjects, (BMI 25.0-29.9 $\mathrm{kg} / \mathrm{m}^{2}$ ), and 142 normal weight patients (BMI 18.5-24.9 kg/m²) The follow-up period was 1 year. The end-point was multiple adverse cardiac events (myocardial infarction, cardiac death, cardiac hospitalization, or emergency department visit). No adverse cardiac events were observed during the follow-up period. Khan and co-workers performed DSE on 555 African American patients. ${ }^{18}$ Intravenous contrast was used when non-contrast images of LV segments were poorly-visualized. There were 409 obese

\section{Alpert}

and 146 non-obese subjects. Multivariate analysis showed that myocardial scar independently predicted allcause mortality and multiple adverse cardiac events. There were no significant differences in these outcomes between obese and non-obese groups. In a recently-published case-control study these outcomes between obese and non-obese groups. In a recently-published case-control study of 1018 patients with a BMI > $35 \mathrm{~kg} / \mathrm{m}^{2}$ who had suffered prior myocardial infarction and who were followed for a median of 4.6 years, Naislund et al compared long-term CV outcomes in 509 patients undergoing metabolic (bariatric) surgery (predominately Roux-en-Y gastric bypass) and 509 patients with severe obesity (BMI [?] $40 \mathrm{~kg} / \mathrm{m}^{2}$ ) who did not undergo metabolic surgery. ${ }^{19}$ Primary outcomes were all-cause deaths and re-admission for myocardial infarction. Secondary outcomes were ischemic or hemorrhagic stroke. Kaplan-Meier analysis showed that metabolic surgery was associated with significantly higher myocardial infarction- and stroke-free survival at 8 years compared to non-surgical controls. Those undergoing metabolic surgery also experienced significantly fewer re-admissions for new-onset heart failure compared to controls. In another recently-published propensity score matched cohort study of 2638 patients with prior CV disease followed for a median of 4.6 years, Doumouras and colleagues reported CV outcomes in 1319 patients with class II and class III obesity undergoing bariatric surgery and 1319 patients who did not undergo bariatric surgery. ${ }^{20} \mathrm{BMI}$ thresholds were $>35 \mathrm{~kg} / \mathrm{m}^{2}$ with a co-morbidity or [?] $40 \mathrm{~kg} / \mathrm{m}^{2}$ without a co-morbidity. Patients receiving bariatric surgery had significantly lower incidence values for multiple adverse CV events (all-cause mortality, myocardial infarction, coronary revascularization, cerebrovascular events, and heart failure hospitalizations compared to those of patients who did not undergo bariatric

Alpert

surgery. Similar results were noted for a 3-component multiple adverse CV event model (all-cause mortality, 
myocardial infarction and ischemic stroke.

Our study differed from previous studies evaluating transthoracic stress echocardiography in severely-obese patients in multiple ways. Six of the previous 9 studies were retrospective and relied heavily on chart review or registry information. ${ }^{14,16-19}$ Our study was prospective and provided a head-to-head comparison of patients with class III obesity receiving intravenous contrast with those who did not receive intravenous contrast. In prior studies, intravenous contrast was used only when baseline non-intravenous contrast images were unsatisfactory except for the study by $\mathrm{Hu}$ et al which focused on overweight and mildly obese patients. ${ }^{12-14,16-18}$ The presence of poor- or non-visualization of [?]2 LV segments, a marker of reduced diagnostic accuracy and interpretability $^{21}$, occurred with significantly greater frequency in the group that did not receive intravenous contrast group than in the group that received intravenous contrast. In previous studies, ESE or DSE were performed without regard as to whether patients had a history of chest pain or pre-existent CAD. ${ }^{13-18}$ In our study, we excluded patients with chest pain or pre-existent CAD. We did so to answer the question whether screening lower risk patients with class III obesity for myocardial ischemia was necessary prior to elective low-risk surgery. Our results suggest that they do not require routine screening for ischemia. Regarding feasibility, several prior studies provided the number of patients who received intravenous contrast, but did not cite the precise criteria for these decisions. ${ }^{13,14,17}$ We not only provided the numbers and percentages of segments well-visualized and interpretable in the intravenous contrast and non-intravenous contrast groups, but also provided a segment-by-segment analysis to determine which LV segments were most likely to be poorly-visualized and

Alpert

not interpretable in both groups. Previous studies performing ESE and DSE and did not comment on whether outcomes differed based on the technique that was chosen. ${ }^{13,14,17}$ Finally, in 7 of the 9 previous studies, the BMI threshold for entry into the study was $<40 \mathrm{~kg} / \mathrm{m}^{2}$ which indicates that not all patients studied were class III obese patients. ${ }^{13,14,16-18}$ The BMI in all of our patients was [?] $40 \mathrm{~kg} / \mathrm{m}^{2}$.

\section{LIMITATIONS}

There are several study limitations. In prior studies, follow-up was often [?] 1 year. Our study provided follow-up at 6 months. Our study was performed prior to the availability of the newest harmonic imaging technology which could further improve endocardial edge detection and reduce the need for intravenous contrast. ${ }^{22}$ We chose the 16 -segment model to assess LV regional wall motion which was recommended by the American Society of Echocardiography at the time that this study was performed. The American Heart Association currently recommends the 17 -segment model, adding the atrial cap to the 16 -segment model. Since our sonographers were instructed to use the 16-segment model at the time of the study, we chose to utilize this model in our analysis. Since we did not routinely perform coronary angiography on the patients in our study, we are unable to provide sensitivity, specificity, and predictive accuracy values. We acknowledge that the relatively young age and the female predominance of our study population reduced the likelihood of the presence of severe CAD. Our results may or may not be applicable to patients with established CAD with a high incidence of regional wall motion abnormalities. Finally, our study was prospective, but was not prospectively randomized or matched.

Alpert

\section{CONCLUSIONS}

This study confirms that DSE is feasible can be performed safely in patients with class III obesity. Our results indicate that DSE with use of IV contrast enhancement is associated with significantly greater visualization and interpretability of LV myocardial segments than DSE without use of IV contrast enhancement. Our study was not designed to assess long-term CV outcomes. However, the low incidence of myocardial ischemia during DSE and absence of deaths and CV morbidity during and 6 months after bariatric surgery suggest that routine stress testing may not be necessary in patients at relatively low CV risk based on a medical history, physical examination, and 12-lead electrocardiogram. 
Alpert

\section{CONFLICT OF INTEREST STATEMENT}

All authors declare that they have no conflicts of interest to report.

\section{AUTHOR CONTRIBUTIONS}

Carl Carlino, MD: research design; data acquisition, analysis, and data interpretation; drafting and critical review of the paper; approval of the submission

Saravanan Kuppuswamy, MD: research design; data acquisition, analysis, and interpretation; critical review of the paper; approval of the submission

Lerin McCray, MD: data analysis and interpretation; drafting the paper; approval of the submission

Kul Aggarwal, MD: data analysis and interpretation; drafting the paper; critical review of the paper; approval of the submission

Martin A. Alpert, MD: supervision; research design; data acquisition, analysis, and interpretation; drafting the paper; critical revew of the paper; approval of the submission

\section{DATA AVAILABILITY}

Data are available upon request forwarded to the corresponding author

FUNDING STATUS: This research was unfunded.

Alpert

\section{REFERENCES}

1. Katta N, Loethen T, Lavie CJ, et al:. Obesity and coronary heart disease: Epidemiology, pathology, and coronary artery imaging.Curr Probl Cardiol. 2020Jul22:100655.doi: 10.1016/j.cpcardiol.2020.100655.

2. Pasternak RC, Grundy SM, Levy D, et al. $27^{\text {th }}$ Bethesda Conference: Matching the intensity of risk factor management with hazard for coronary disease events. Task Force 3. Spectrum of risk factors for coronary heart disease. J Am Coll Cardiol. 1996:27(5):978-990.

3. Gallagher MJ, Franklin BA, Ehrman SK, et al: Comparative impact of morbid obesity vs. heart failure on cardiorespiratory fitness. Chest. 2005:127(6):2197-2204.

4. Catheline JM, Bihan H, LeQuan TC,et al. Pre-cardiac and pulmonary evaluation in operative bariatric surgery. Obes Surg. 2008:18(3):271-277.

5. El Hajj MC, Litwin SE. Echocardiography in the era of obesity. J Am Soc Echocardiogr. 2020:33(3):779789 .

6. Ahmed H. Contrast echocardiography: Clinical applications. www.uptodate.com. Accessed on December $14,2020$.

7. Shah BN, Senior R. Stress echocardiography in patients with morbid obesity. Echocardiography Research and Practice. 2016: 3(2): R13-18.

8. World Health Organization Technical Report 894. Obesity: Preventing and managing the global epidemic. World Health Organization, Geneva, 2000.

Alpert

9. Pellikka PA, Arruda-Olsen A, Chaudry FA, et al:.Guidelines for performance, interpretation, and application of stress echocardiography in ischemic heart disease. J Am Soc Echocardiogr. 2020:33(1):1-41. 
10. Porter TR, Mulvagh SC, Abdemoneim SS, et al: Clinical application of ultrasonic enhancing agents in echocardiography. J Am Soc Echocardiogr. 2018:31(3):271-274.

11. Cerqueira MD, Weissman NJ, Dilsizian V, et al:.Standard myocardial segmentation and nomenclature for tomographic imaging of the heart. Circulation. 2002:105(4):539-542.

12. Lerakis S, Kalogeropoulos AP, El-Chami MF, et al: Transthoracic dobutamine stress echocardiography in patients undergoing bariatric surgery. Obes Surg. 2007:17(11):1475-1478.

13. Shah BN, Zacharius K, Pabla JS, et al. The clinical impact of contemporary stress echocardiography in morbid obesity for the assessment of coronary artery disease. Heart. 2016:102(5):370-375.

14. Supariwala A, Makani H, Kahan J, et al: Feasibility and prognostic value of stress echocardiography in obese, morbidly obese, and super-obese patients requiring bariatric surgery. Echocardiogr. 2014: 31(7):879885.

15. Hu SJ, Liu SX, Katus M, Luedde M. The value of dobutamine stress echocardiography in detecting coronary artery disease. Canad J Cardiol. 2007:23(11):885-889.

16. Siviera MG, Sousa AC, Santos MA, et al:. Assessment of myocardial ischemia in obese individuals undergoing physical stress echocardiography (PSE). Arq Bras Cardiol. 2015:104(5):394-400.

Alpert

17. Murphy M, Krothapalli S, Cuellar J, et al: Prognostic value of normal stress echocardiography in obese patients. J Obesity. http://dx.doi.org/10.1155/2014/419724. EpubAug31.

18. Khan A, Valdiviezo E, Singh G, et al: Prognostic utility of dobutamine stress echocardiography in obese African American population. Open J Med Imaging. 2012: 2:50-56.

19. Naislund E, Stenberg E, Hofmann R, et al: Association of meta bolic surgery with major adverse cardiovascular outcomes in patients with previous myocardial infarction and severe obesity: A nationwide cohort study. Circulation. 2021:143(15):1458-1467.

20. Doumouras AG, Wong J, Paterson JM, et al: Bariatric surgery and cardiovascular outcomes in patients with obesity and cardiovascular disease: A population-based retrospective cohort study. Circulation. 2021:143(15):1468-1480.

21. Plana JC, Mikati IA, Dokainch H, et al: A randomized crossover study for evaluation of the effect of image optimization with contrast on the diagnostic accuracy of dobutamine echocardiography in coronary artery disease. The OPTIMIZE trial. J Am Coll Cardiol: Cardiovasc Imaging 2008:1(2):145-152.

22. Hyun D, Crowley ALC, LeFevre M, et al: Improved visualization in difficult-to-image stress echocardiography patients using real-time harmonic spatial coherence imaging. IEEE Trans Ultrasonic Ferroelectric Freq Control. 2018:6 